\title{
Hype oder langfristiger Trend?
}

\author{
Ulrich Schartow
}

Mit der Einführung von Marktmechanismen im Sozialbereich wurde die Praxis der Investitionsfinanzierung durch öffentliche Zuschüsse eingeschränkt oder abgeschafft. Der daraus resultierende Finanzbedarf sozialer Träger trifft auf weltweit vorhandene enorme Kapitalmengen, die wegen der lang andauernden Niedrigzinsphase auf der Suche nach attraktiven Anlagemöglichkeiten sind. Angesichts der demografischen Entwicklung interessieren sich deshalb viele Anleger insbesondere für Investitionen in Pflegeimmobilien.

Innerhalb der vergangenen Monate verging nahezu keine Woche, in der nicht mindestens eine Fondsgesellschaft ihren Einstieg in das Segment der Sozialimmobilien ankündigte oder über bereits vollzogene Transaktionen informierte. Sind bereits die gehandelten Volumina beeindruckend bis Ende August 2006 zirka 750 Millionen Euro -, so führt die Summierung des von Investorenseite als zur Verfügung stehend deklarierten Kapitals schnell zu Milliardenbeträgen, die direkt in den Markt investiert werden können.

Goldene Zeiten also für die Eigentümer von Sozialimmobilien, wenn Marktteilnehmer »eskalierende Kaufpreise« konstatieren - oder doch nicht? Neben dieser Frage soll im Folgenden insbesondere erläutert werden, wo die Ursachen für den aktuellen Nachfragesog liegen, welche Anforderungen an den Zugang zu Investorenkapital geknüpft werden, ob der Status der Gemeinnützigkeit spezifische Besonderheiten mit sich bringt und unter welchen Voraussetzungen die Fondsfinanzierung für die Beteiligten auch auf Dauer attraktiv sein kann.

\section{Ursachen des Anlegerinteresses}

Das gegenwärtig sehr ausgeprägte Interesses an Investments im Segment der Sozialimmobilien in Deutschland geht zunächst einmal auf den im Verlauf des letzten Jahrzehnts politisch initiierten Richtungswechsel hin zu einer Ökonomisierung der Sozialarbeit allgemein und damit auch der Finanzierung von Sozialimmobilien zurück. Durch die Einführung von Marktmechanismen sollen die Vorteile einer effektiven Ressourcenallokation für die Sozialwirtschaft genutzt werden. In der Folge wurde die jahrzehntelange Praxis der Investitionsfinanzierung durch Zuschüsse massiv zurückgefahren. Hieraus erwächst erst der bis dahin kaum vorhandene Bedarf an Mitteln des freien Kapitalmarktes.

Handelt es sich bei dieser Ursache quasi um eine Veränderung innerdeutscher Rahmenbedingungen, so er-

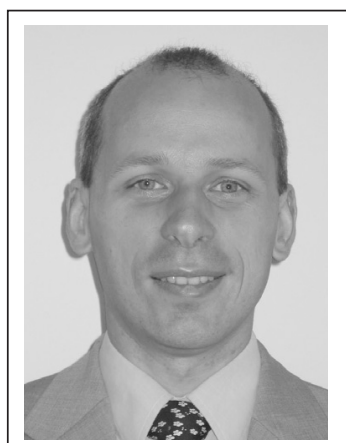

Ulrich Schartow, geboren 1969 in Schwedt (Oder), ist diplomierter Bankbetriebswirt. Er blickt auf eine langjährige Tätigkeit in der Kreditzentrale der Bank für Sozialwirtschaft AG zurück. Seit 2005 ist er Geschäftsführer der IS Immobilien-Service GmbH. Diese befasst sich mit der Entwicklung von Analysetools zur Beurteilung der Realisierungsfähigkeit von Sozialimmobilien, mit der konzeptionellen Beratung sozialwirtschaftlicher Organisationen bei der Erschließung neuer Standorte und beim Einsatz alternativer Finanzierungsinstrumente.

schließt sich der Zustrom von Fremdkapital in den zuvor genannten Größenordnungen nur durch einen Blick auf die internationalen Finanzmärkte. Bedingt durch eine in der Historie der Dauer als auch den absoluten Werten nach bisher nicht gekannte Niedrigzinsphase sind weltweit enorme Kapitalmengen auf der Suche nach Anlagemöglichkeiten, die eine höhere Rendite als die gewöhnlichen Staatsanleihen versprechen. Da die größte Kapitalkonzentration traditionell bei Vermögensverwaltern des angelsächsischen Raums liegt, treten diese im Fondsbereich in der Folge auch am häufigsten als Investor in Erscheinung. Selbstverständlich sind auch die traditionellen deutschen institutionellen Anleger wie Banken, Versicherungen und Pensionskassen sowie vermögende Privatanleger am Markt aktiv, sodass Liquidität grundsätzlich nicht das Problem ist. Doch worauf zielen Investoren genau ab, wenn sie ihr Interesse an Sozialimmobilien bekunden?

\section{Sozialimmobilie ja, Renditeverzicht nein}

Wie bereits erläutert, wird das Anlegerinteresse grundsätzlich von Renditeerwartungen bestimmt. Aspekte, die der Investition Attribute in Richtung einer ethischen Geldanlage verleihen, sind ein willkommenes Plus, begründen aber in der Regel keine Anlageentscheidung. Jeder Träger von Sozialimmobilien mit Bedarf an Fremdkapital sollte sich dieser Ausgangssituation bewusst sein.

Aufgrund der gegenwärtigen Kapitalmarktopportunitäten ist das Renditeziel nicht unter sechs Prozent zu beziffern. Hierbei ist zu berücksichtigen, dass der Ertrag der 
heutigen Fonds aus dem Cash Flow tatsächlich erwirtschaftet werden muss. Denn nach der Streichung der Steuervorteile in Form von Verlustzuweisungen, die der Konstruktion der meisten Fonds in den 1990-Jahren zu Grunde lag, stehen jetzt ausschließlich die aus den Immobilien in Form der Pachtzahlungen zurückfließenden Erträge zur Erreichung der erstrebten Anlageziele zur Verfügung.

In den Fokus der Anleger rücken damit nur die Sozialimmobilien, die solche Renditeerwartungen erfüllen können. Dies führt dazu, dass der Sammelbegriff Sozialimmobilie sich in der konkreten Umsetzung auf die Pflegeimmobilie verengt. Zurückzuführen ist dies auf die in diesem Segment im Vergleich zu anderen wie beispielsweise Werkstätten für behinderte Menschen oder Einrichtungen der Kinder- und Jugendhilfe am weitesten fortgeschrittene Marktöffnung und Refinanzierungsbedingungen, die den genannten Renditeerwartungen entgegen kommen. Daher bewegen sich Sozialimmobilienfonds nahezu ausschließlich im Bereich der stationären Pflege, womit eine Bezeichnung als Pflegeimmobilienfonds ihren Inhalt weitaus präziser beschreiben würde. Da gerade bei gemeinnützigen Trägern die stationäre Pflege in der Regel nur einen Teil ihres Engagements an Sozialer Arbeit ausmacht wird deutlich, dass Sozialimmobilienfonds nicht die Pauschallösung für die Investitionserfordernisse des gesamten Immobilienportfolios darstellen können.

\section{Aufbau von Überkapazitäten}

Auch wenn der Pflegemarkt in Deutschland stark fragmentiert ist, stellen die gemeinnützigen Träger der Freien Wohlfahrtspflege in der Summe traditionell das Gros der Pflegeplätze - noch. Denn das aktuelle Wachstum an neuen modernen Platzkapazitäten entfällt zum ganz überwiegenden Teil auf private Anbieter. Setzt sich diese Tendenz weiterhin ungebremst fort, ist abzusehen, dass die Freie Wohlfahrtspflege in absehbarer Zeit deutlich in die Defensive gedrängt wird. Ein wesentlicher Grund für diese Entwicklung ist das Erwartungsparadoxon der Anbieter, das Triebfeder der massiven Neubautätigkeit ist (vgl. Grafik).

Der Ausbau der Platzkapazitäten folgt im Wesentlichen der Argumentationskette, dass aus der unbestreitbaren Steigerung der Anzahl Hochaltriger in den kommenden Jahrzehnten ein in der Vergangenheit beobachteter beinahe paralleler Anstieg des stationären Pflegebedarfs auch für die Zukunft abgeleitet werden könne. Die gegenwärtige Popularität von Pflegeheimen bei Investoren ist maßgeblich hierauf zurückzuführen, lässt sich damit doch die Aussicht auf sicheren und zudem bequem erzielbaren Gewinn nähren.

Die in der Folge gegenwärtig zum Teil im Raum stehenden Kaufpreisfaktoren erreichen dabei Höhen, die Merkmale für durch einen Hype hervorgerufene Marktüberhitzung aufweisen. Während die Annahme hinsichtlich der Alterung der Bevölkerung zweifelsfrei begründet werden kann, beruht die Schlussfolgerung hinsichtlich der notwendigen Pflegeplatzkapazitäten auf Prognosen, für die es statistisch keine Grundlage gibt.
Die aktuelle Pflegestatistik des statistischen Bundesamtes weist nämlich nach, dass sich die Zahl der tatsächlich Pflegebedürftigen aus verschiedenen Gründen nur deutlich unterproportional zur Alterung der Bevölkerung entwickelt. Das paradoxe dieser Situation besteht darin, dass die so bereits geschaffenen oder zu erwartenden Überkapazitäten weitestgehend nicht zu Lasten der Neubauplätze gehen, sondern eine Verschärfung der Wettbewerbssituation hervorrufen, die zu einer Verdrängung alter und nicht mehr marktgerechter Plätze führt. Denn entgegen mancher Annahme verteilt sich ein Überhang an Plätzen eben nicht gleichmäßig auf die vorhandenen Anbieter. Vielmehr führt der Marktmechanismus zu Wettbewerbsgewinnern mit vollen Häusern und Wettbewerbsverlierern mit Existenz bedrohenden Leerständen.

\section{Bedeutung der Fondsfinanzierung}

Mit der zuletzt beschriebenen Herausforderung konfrontiert sehen sich alle die Träger, die in ihrem Bestand veraltete und somit auf Dauer nicht marktfähige Pflegeimmobilien aufweisen. Eine Reaktion auf diese Entwicklung kann nicht bis zum tatsächlichen Eintritt von Belegungsrückgängen aufgeschoben werden, da von der Einleitung von Sanierungs- oder Neubaumaßnahmen bis zu deren Abschluss zumeist zwei bis drei Jahre vergehen. In einem solchen Zeitraum können schnell Verluste auflaufen, die den Fortbestand eines Trägers insgesamt in Frage stellen.

Die erfolgreiche Positionierung im Markt wird also auch für etablierte Betreiber nur möglich sein, wenn diese kontinuierlich in den Immobilienbestand investieren. Für eine klassische Darlehensfinanzierung bedarf es zumeist eines Eigenkapitalanteils von in der Regel 20 Prozent. Theoretisch müssten diese Mittel auch zur Verfügung stehen, denn schließlich beinhaltet der vergütete Investitionskostensatz einen Anteil für die Instandhaltung, der grundsätzlich zu separieren ist.

In der Realität jedoch sehen sich viele Organisationen mit der Tatsache konfrontiert, dass die eigentlich für die Instandhaltung vorgesehen Mittel in den allgemeinen Haushalt eingestellt wurden und so nicht mehr zur Verfügung stehen. Die im internationalen Vergleich ohnehin unterdurchschnittliche Eigenkapitalausstattung deutscher Unternehmen gewinnt bei gemeinnützigen Trägern noch zusätzliche Brisanz dadurch, dass den Vorteilen dieses Status erhebliche Einschränkungen bei den Möglichkeiten der Eigenkapitalbildung entgegenstehen. Der Zugang zu Fremdkapital wird dadurch erheblich erschwert.

Vorrangige strategische Zielsetzung muss es deshalb sein, die vorhandenen Eigenkapitalressourcen optimal auszuschöpfen. Eine seit langem etablierte Methode vorhandenes Eigenkapital freizusetzen besteht im Verkauf und gleichzeitiger Rückmietung vorhandener Immobilien als sogenanntes Sale-and-lease-back. Der Verkauf einzelner Objekte an Immobilienfonds stellt in diesem Zusammenhang eine sehr gute Möglichkeit dar, die Kapitalstruktur der eigenen Organisation durch Hebung eventuell vorhandener stiller Reserven im Anlagevermögen einerseits und durch Zuführung von liquiden Mitteln andererseits $\mathrm{zu}$ verbessern. Insofern ist die gegenwärtige 


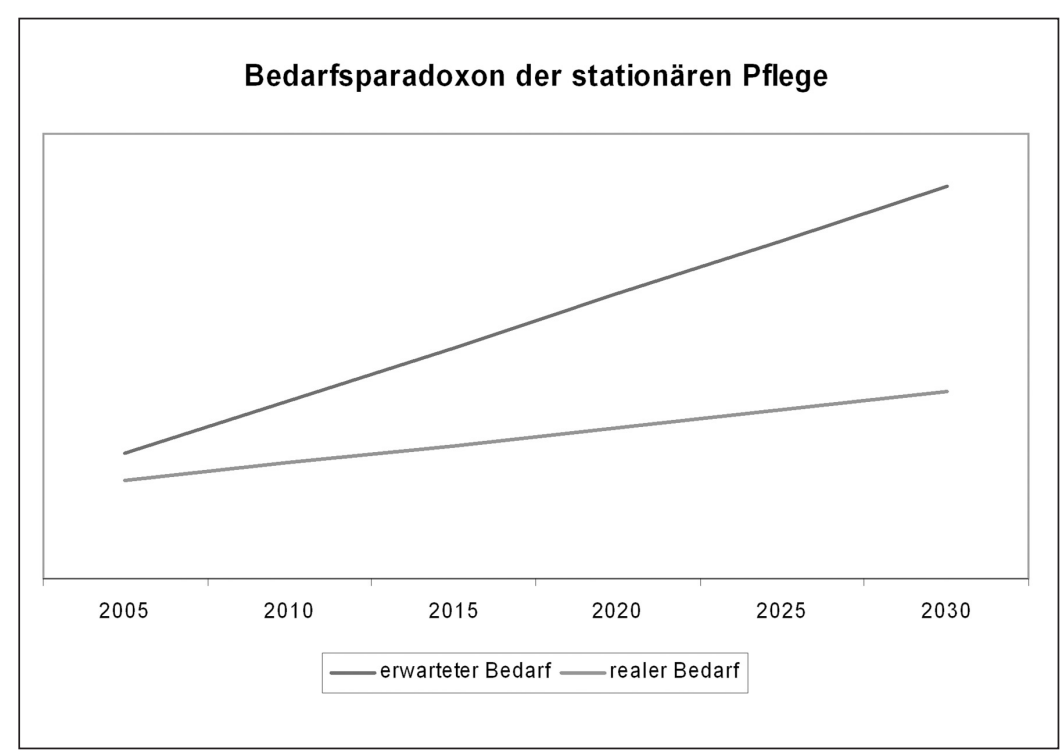

Marktsituation zumindest für die Eigentümer von Pflegeimmobilien wenn schon nicht golden, wie eingangs apostrophiert, so doch äußerst günstig, um attraktive Verkaufspreise zu verhandeln. Doch auch unabhängig von aktuellen Marktanomalien sollte die Erörterung der Möglichkeit einer Fondsfinanzierung zum Standardrepertoire von Pflegeheimeigentümern gehören. Insofern ist dieser Finanzierungsform grundsätzliche Bedeutung beizumessen.

\section{Anforderungen der Investoren}

Die Renditeerwartung als zentrales Element des Investoreninteresses wurde bereits benannt. Im betrachteten Segment ist darüber hinaus die Eigenschaft als Betreiberimmobilie zu berücksichtigen. Dies bedeutet, dass die Nutzung derartiger Gebäude erst durch die Mittlerrolle eines Betreibers zum Endnutzer möglich wird, der in der Regel selbst weder Eigentümer noch Mieter der Immobilie ist.

Der Qualität des Betreibers kommt daher für den Erfolg entscheidende Bedeutung zu. Entsprechend akribisch prüfen Fonds vor einer Kaufentscheidung den Betreiber hinsichtlich seiner Fähigkeit, langfristig eine hohe Auslastung sicherstellen zu können. Fonds erwerben selbst keine Objekte, die von der Gebäudequalität oder ihrer betriebswirtschaftlichen Effizienz her Sanierungsbemühungen notwendig machen. Diese Aufgabe müssen bei Bestandsimmobilien die Voreigentümer oder Betreiber durchführen.

Zum Verständnis der Kalkulation eines Immobilienfonds ist es zudem unumgänglich, sich den direkten $\mathrm{Zu}$ sammenhang zwischen der Ausgestaltung des Pachtvertrages und dem erzielbaren Kaufpreis deutlich zu machen. Da die Angemessenheit von Kaufpreisen überschlägig als Faktor der Pachtzahlung ermittelt wird, steigt dieser kongruent zur Pacht. Im Interesse der Fonds liegt es zudem, den administrativen Aufwand bei der Eigentumsverwaltung möglichst gering zu halten. Fonds erwarten daher, dass die gesamte Instandhaltung der Objekte bis auf Dach und Fach durch die Betreiber übernommen wird. Dieser Aufwand ist von den Betreibern daher zwingend bei der
Verhandlung der Pachthöhe zu berücksichtigen.

\section{Besonderheiten im gemeinnützigen Sektor}

Hinsichtlich der Handhabung der wirtschaftlichen Rahmenbedingungen ergeben sich für gemeinnützige Organisationen keine Besonderheiten. Die Intention des Gesetzgebers zielt ja gerade darauf ab, punktuell aus der Vergangenheit noch bestehende Regelungen, die nicht dem Marktmechanismus entsprechen, vollständig abzubauen. Dass zwischen Immobilienfonds und gemeinnützigen Eigentümern und Betreibern zuweilen Schwierigkeiten im gegenseitigen Verständnis auftreten, liegt daran, dass gemeinnützige Träger besondere Anforderungen hinsichtlich der Langfristigkeit des Engagements und des Verständnisses für die eigene ethisch-inhaltliche Ausrichtung an ihre Finanzierungspartner stellen müssen, die über Aspekte der reinen Finanzkraft deutlich hinausgehen.

In der Folge kann es durchaus zu Komplikationen in der Zusammenarbeit kommen, die etwa daraus resultieren, dass der Fonds seine Mittel ausschließlich auf der Suche nach den günstigsten Kapitalmarktopportunitäten investiert und daher nur einen mittelfristigen Anlagehorizont verfolgt. Für den Betreiber kann es dann schon einen Unterschied ausmachen, wenn er es mit wechselnden Fondsgesellschaften zu tun bekommt. Potenziert wird diese Problematik durch die räumliche Entfernung der jeweiligen Entscheidungsträger vom deutschen Markt. Langfristig erfolgreich wird die Zusammenarbeit von Immobilienfonds und gemeinnützigen Organisationen daher nur dann sein, wenn die Identifikation mit dem Sozialmarkt von beiden Seiten als auf Dauer angelegt betrachtet wird.

\section{Ausblick}

Dem Verlauf jedes Marktzyklus folgend, wird sich auch der gegenwärtig festzustellende Nachfrageüberhang auf der Fondseite wieder normalisieren. Spätestens dann, wenn das Zinsniveau seinen langsamen Aufwärtstrend fortsetzt.

Neben den klassischen Immobilienfonds wird das Jahr 2007 wahrscheinlich die immer wieder verschobene Einführung des Real Estate Investment Trust (Reit) bringen. Mit dieser besonderen Form einer Immobilienaktiengesellschaft steht dem Immobilienmarkt in Deutschland dann ein international bereits erprobtes Investitionskonstrukt zur Verfügung.

Die Anforderungen eines erfolgreichen Engagements in Pflegeimmobilien ändern sich hierdurch jedoch nicht, denn die zentrale Voraussetzung wirtschaftlicher Prosperität bleibt die Qualifikation des Betreibers in Verbindung mit einem langfristig ausgerichteten Interesse des Immobilieneigentümers. 